\title{
Combining the spectral information of Dual-Band images to enhance contrast and reveal details
}

by S. Quirin* and H.-G. Herrmann**

\author{
* Chair for Lightweight Systems, Saarland University, Campus E3 1, 66123 Saarbrücken, Germany, \\ steven.quirin@uni-saarland.de \\ ** Fraunhofer Institute for Nondestructive Testing and Chair for Lightweight Systems, Campus E3 1, \\ 66123 Saarbrücken, Germany
}

\begin{abstract}
A method to calculate an emissivity image out of two thermographic images taken at different wavelengths has been developed. Main part is a thermal pixel to pixel calibration to make measurements physically comparable. The new image shows enhanced contrast in several nondestructive testing situations and reveals details that could not be accessed in the input images. Examples for passive and active thermography are presented using an infrared dual-band camera working simultaneously in the MWIR and LWIR band. Reflection characteristics of CFRP and its components apparent in thermography have been compared with reflectance spectroscopy measurements.
\end{abstract}

\section{Introduction}

Different materials and surfaces have a very characteristic interaction with infrared radiation. Polymers have narrow absorption bands that are characteristic for their chemical composition. High absorption in certain regions of the infrared spectrum can also be found in materials like ceramics and glasses. Metallic elements and alloys show a more monotonic but also non-linear absorption behaviour. Materials can be distinguished very well by analysis of their infrared spectrum with Infrared Spectrometers. Depending on the realization this is at least a time consuming process (Thermal Infrared Multispectral Scanner, TIMS) or not giving imaging information. As thermographic image detectors are broad band detectors, a band analysis can be realized by the use of different filters in a rotating filter wheel. In this research, a dual-band camera is used instead to avoid tilt of filters in the wheel [1] and time difference between measurements until the filter is changed resulting in pixel maps that do not correspond to the same position and state of the sample. Temperature change of filters between calibration and measurement also affects detected radiance and can be avoided since dual band detector is cooled as a whole. Another advantage could be, that wavelengths lie more apart from each other as different detector materials are used, so infrared characteristics differ more significant from each other when they change monotonic [2]. Analysis is done in several testing situations like passive, flash and induction thermography. As test object, a 2.5-dimensional carbon fibre reinforced plastic (CFRP) is used (figure 2a). Reflectance spectroscopy measurements are conducted to support the observation that infrared optical properties of CFRP are highly wavelength dependant.

\section{Band analysis and calibration method}

Concluding from radiation balance, the sum of absorption, reflection and transmission follows the function

$$
\alpha+\rho+\tau=1
$$

Transmittance of CFRP sample proved to be negligible in own measurements and literature [3]. Kirchhoff's Law is taken to replace $\alpha$ by emissivity $\varepsilon$ to get the relationship

$$
\rho=1-\varepsilon
$$

Detected radiation consists of the sample surface's spectral radiance $L$ at temperature $T$ and its reflection of ambient heat $T_{a}$ according to Eq. (3) following Planck's law Eq. (4). In case of an optical imaging system, irradiance $E$ for every pixel is calculated using Eq. (5). Hence, the total radiance $L_{\text {total }}$ is integrated over the sensitivity range of the infrared detection $\left(\lambda_{1}\right.$ to $\left.\lambda_{2}\right) . c_{1 L}$ and $c_{2}$ summarize physical constants according to international temperature scale (ITS90).

$$
L_{\text {total }}(\lambda, T)=\varepsilon(\lambda) \cdot L(\lambda, T)+(1-\varepsilon(\lambda)) \cdot L_{a}\left(\lambda, T_{a}\right)
$$




$$
\begin{aligned}
& L(\lambda, T)=\frac{c_{1 L}}{\lambda^{5}} \frac{1}{\exp \left(\frac{c_{2}}{\lambda T}\right)-1} \\
& E(T, \varepsilon)=\int_{\lambda_{1}}^{\lambda_{2}} \varepsilon(\lambda) \cdot \frac{c_{1 L} L}{\lambda^{5}} \cdot \frac{1}{\exp \left(\frac{c_{2}}{\lambda T}\right)-1}+(1-\varepsilon(\lambda)) \cdot \frac{c_{1 L}}{\lambda^{5}} \cdot \frac{1}{\exp \left(\frac{c_{2}}{T_{a}}\right)-1} d \lambda
\end{aligned}
$$

Thermographic systems are used in field where temperature of the specimen is not uniform. Local temperature distribution on the specimen highly affects quantity of radiation, thus generating the contrast in unprocessed thermographic images. Therefore, processing to get an emissivity image is proposed [2,4]. Processing here implies a simple ratio of the irradiance in different measured wavelength bands, i.e. the mid-wavelength infrared (MWIR) and the long-wavelength infrared (LWIR).

$$
E_{\text {Result }}=\frac{E_{\lambda_{A}}}{E_{\lambda_{B}}}=\frac{E_{M W I R}}{E_{L W I R}}
$$

Main obstacle to apply this calculation is that a physical correlation between images in terms of quantity of measured irradiance is not given by camera manufacturers in both cases of a multi-band detector or band-pass filters. Even the temperature calibration procedure is often not satisfactory [5]. Backward calculation from temperature to irradiance can only be solved numerically and is time consuming anyway. A calibration method to get intensity of radiation for every pixel in the thermographic images is needed. The developed method is based on Planck's law according to Eq. (5). A polynomial calibration curve as it is used by Pron and Bouache [5] could also be imagined. But the polynomial function needs more than two fitting variables and therefore as much calibration points to determine them. However, the proposed method only needs two calibration points whereas one can be taken at room temperature. A black body radiation source is used to get radiance at two different temperatures in the considered wavebands. The infrared detector is a photon counting QWIP detector, thus the output is linear to irradiance in a wide measurement region [6] proved in figure 1c. The calibration curve is based on a simple linear relation between the two calibration points. Calculation has been optimized taking into account non-ideal optical properties of blackbody radiation source (emissivity) and detector (spectral sensitivity curve [1]) by precalculated correction factors suitable for any measurement. Accuracy of calibration method turned out to be much better if interpolated than if extrapolated to temperatures lying outside calibration range. LWIR range is reproduced much better than MWIR range, but the method showed sufficient accuracy for the calculation of emissivity image for enhanced contrast in experiments.

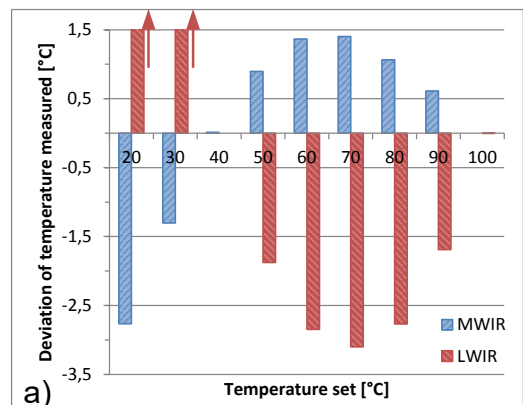

Fig. 1. Accuracy of calibration method for calibration at 40 and $100{ }^{\circ} \mathrm{C}$. Measured irradiance has been calculated back to temperature numerically to compare with real temperature of blackbody calibration source. Calibration method a) based on unmodified Planck's law, b) optimized, c) camera raw output linear to irradiance

\section{Experiments}

The experiments are performed using Thermosensorik QWIP Dualband 384, an infrared dual-band camera working simultaneously in the 4.4-5.2 $\mu \mathrm{m}$ (denoted as MWIR) and the 7.8-8.8 $\mu \mathrm{m}$ (denoted as LWIR) bands. A frame rate of $146 \mathrm{~Hz}$ is possible at standard resolution of 384 x 288 px. A Mikron Infrared M345X4 blackbody source has been used for calibration. The camera detector is cooled by a sterling-type cooler. Camera housing serves as a heat exchanger and reaches a temperature about $40{ }^{\circ} \mathrm{C}$ which can be seen as a reflection on a cold sample. The sample is a 2.5-dimensional CFRP because it consists of a metal-polymer insert that is placed in between CFRP layers during Resin Transfer Moulding Process [7]. Several examples for experimental results of non-destructive testing situations are given in this paper. 


\subsection{Induction Thermography}

A coil is placed behind the sample to excite it with heat periodically. Images are taken around the peak of contrast of the heat cycle stated below (figures $2 \mathrm{~b}, 3$ ). Temperature of the sample rose approximately $2{ }^{\circ} \mathrm{C}$ until $5^{\text {th }}$ heat cycle. The sample used is painted black half side with removable spray film (figure 2a). The distance between camera and sample is $25 \mathrm{~cm}$.

a)
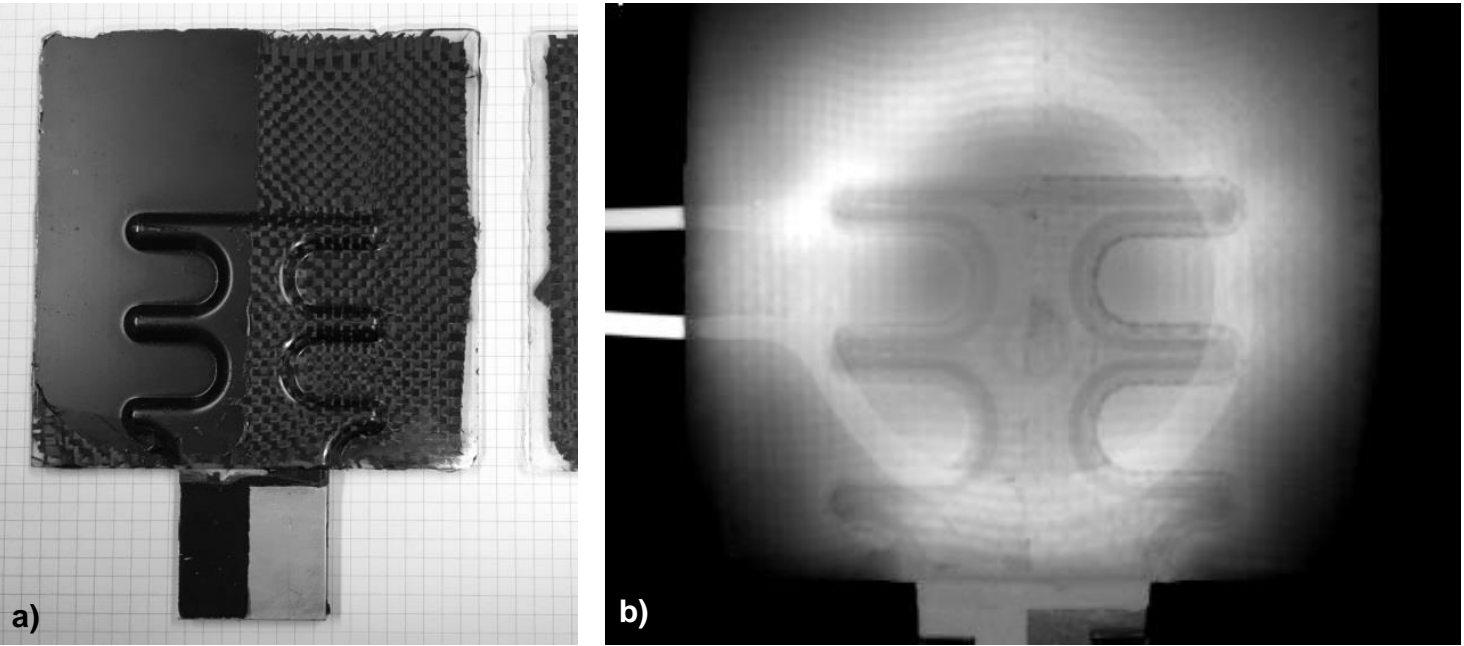

Fig. 2. a) CFRP sample frontside: half side painted (backside: non-painted), b) experimental setup for induction thermography: image of sample after 5 cycles excitation superimposed with image of coil

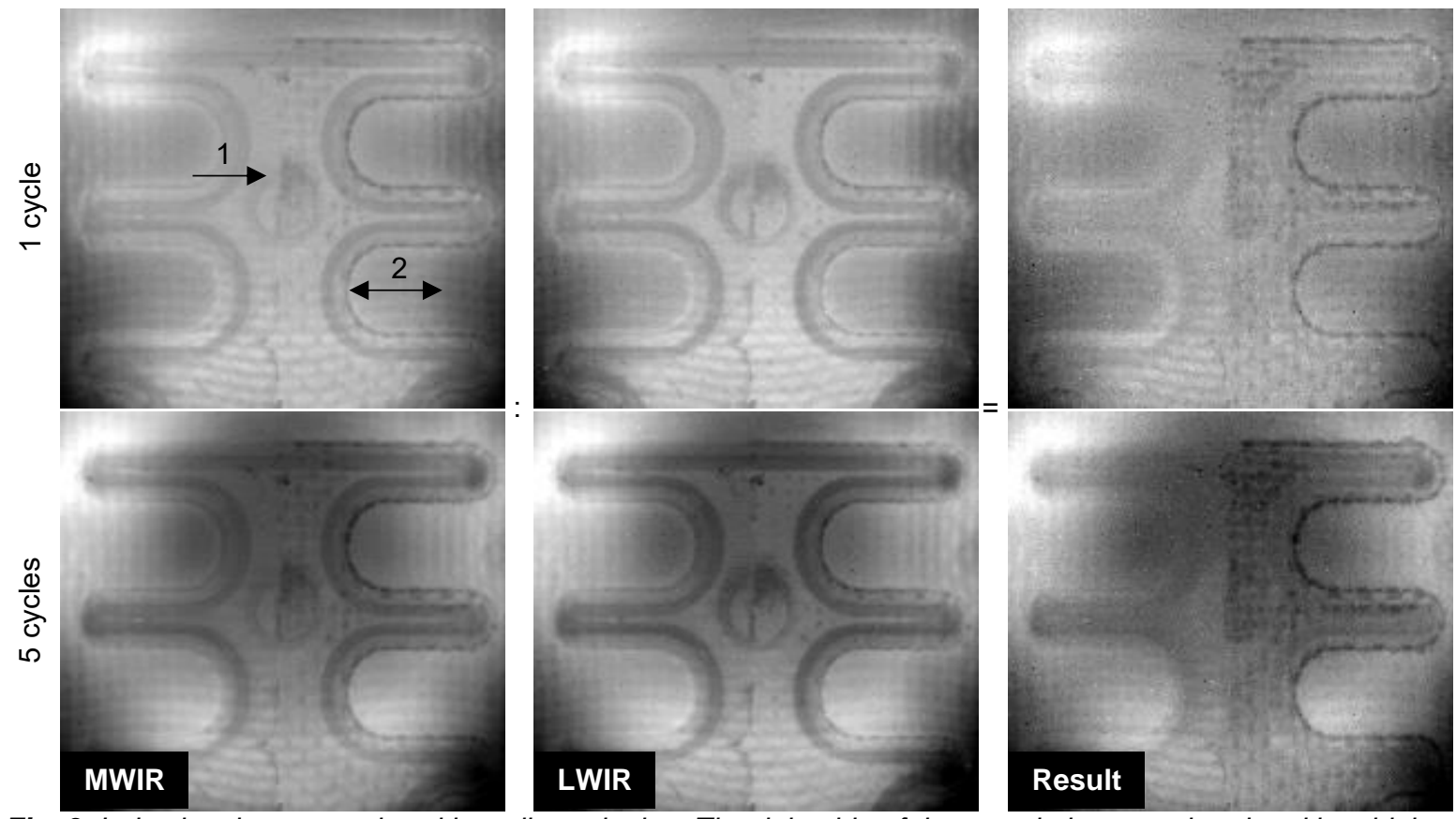

Fig. 3. Induction thermography with cyclic excitation. The right side of the sample is non-painted and has higher reflectance. Cold spot caused by narcissus effect (1) and reflection of camera housing (2) are suppressed in the result as well as 3D structure of the sample. Result image is noisier but shows a structure in non-painted area which is not visible in input images.

\subsection{Flash Thermography}

Flash thermography was carried out to simulate a difficult field thermography measurement where reflections of surroundings appear onto the surface of a sample. The sample is inclined to the optical path of camera and flash in the setup. Sample was painted, spray film around the metal-polymer insert has been removed for measurements shown in figure 4 and 5 . The distance between camera and sample is about $25 \mathrm{~cm}$. 

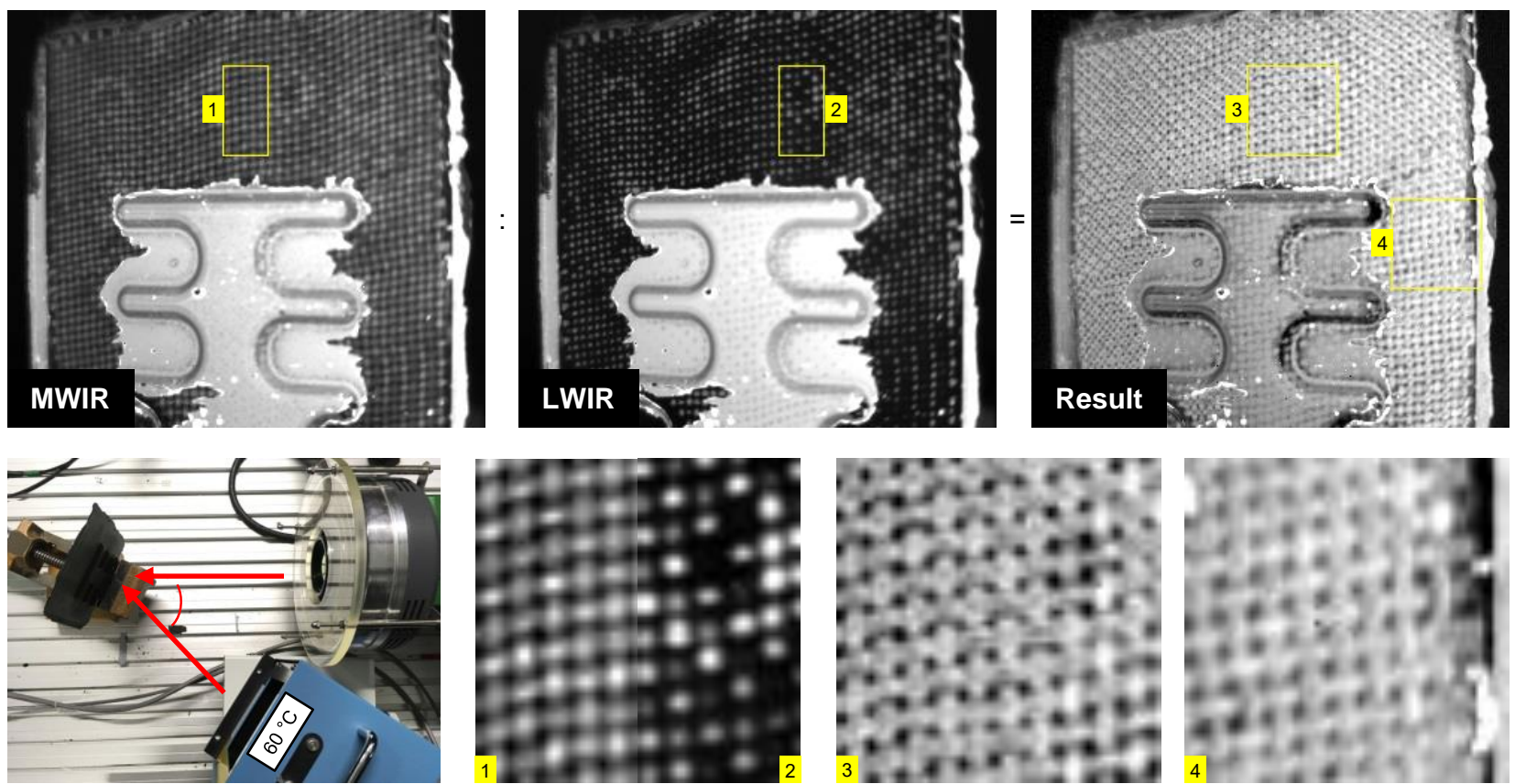

Fig. 4. Flash thermography setup, image taken during flash firing with power of $3.2 \mathrm{~kJ}$, framerate 146 fps. High global contrast between painted and non-painted area was replaced by high local contrast resulting in a highly plastic image of woven fibre structure.
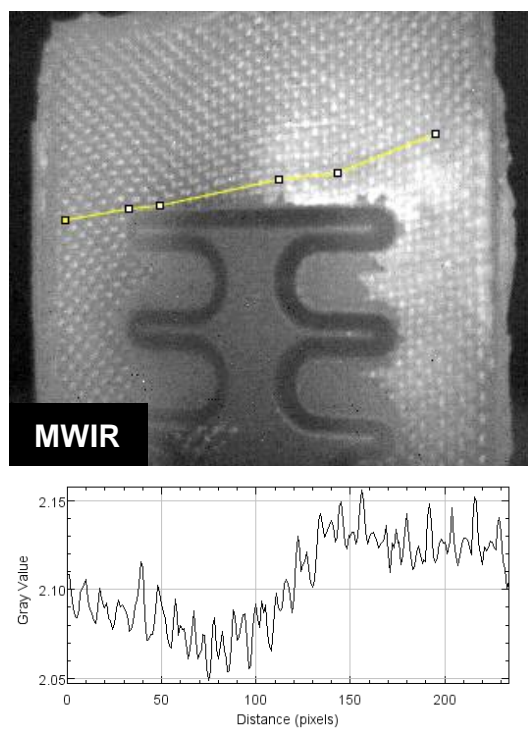
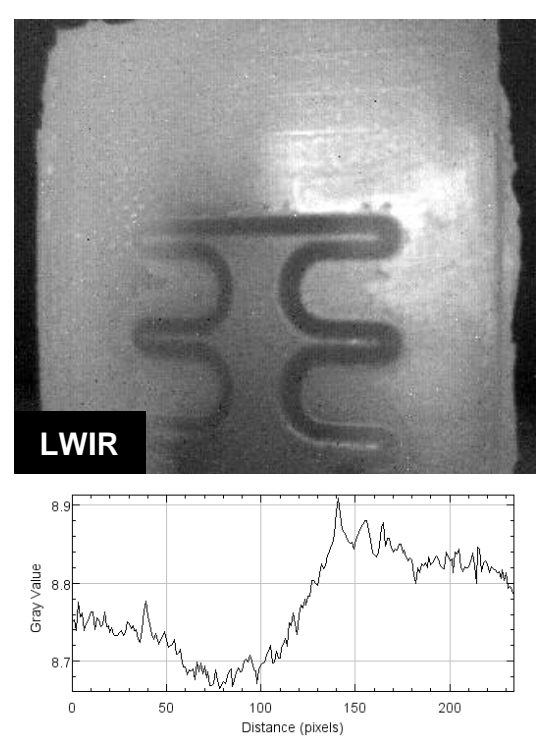
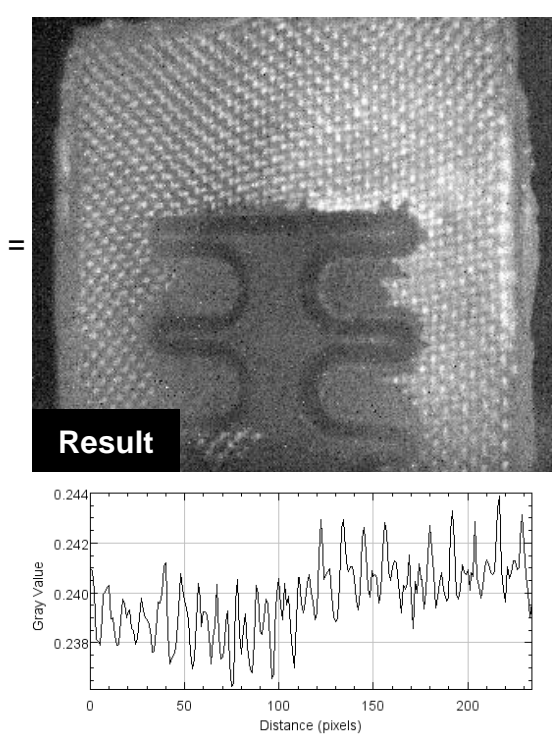

Fig. 5. Flash thermography setup, image taken after dissipated heat of flash faded away, so sample was slightly heated over whole area. Reflection of infrared source is clearly visible in input images. Reflection is suppressed and local contrast increased in the result image. Path following a fibre through the woven fabric.

\subsection{Passive Thermography}

Two results which show the potential of the method are shown below. The first one is a CFRP sample which was heated with induction coil. After heat dissipated, it was recorded in low distance (about $15 \mathrm{~cm}$ ) to the camera. Hence, the camera resolution wasn't a factor decreasing local contrast after processing due to noise. This example shown in figure 6 highlights the observations made above. Reflection of camera housing and of detector, the latter is called narcissus effect, could be suppressed. The other passive thermography situation is not a sample but a snapshot taken from distance of the instrument's operator, showing his T-shirt print (figure 7). 

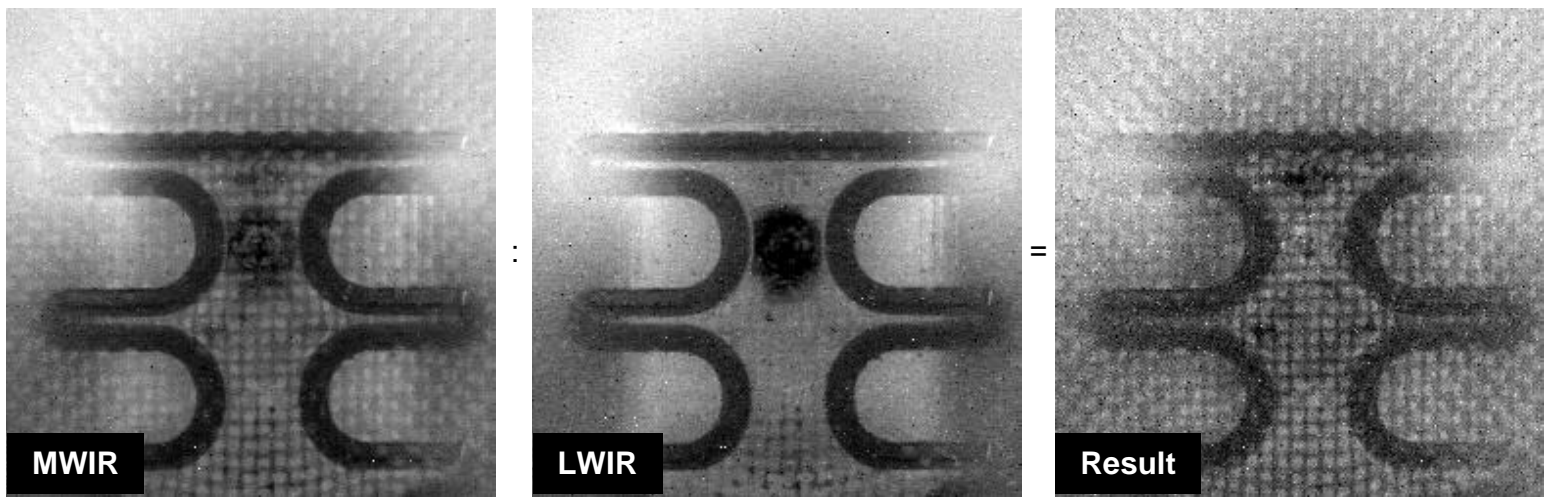

Fig. 6. CFRP structure taken after induction thermography. Reflections (cold spot caused by narcissus effect, camera housing) could be suppressed and details revealed by combining the two input images.
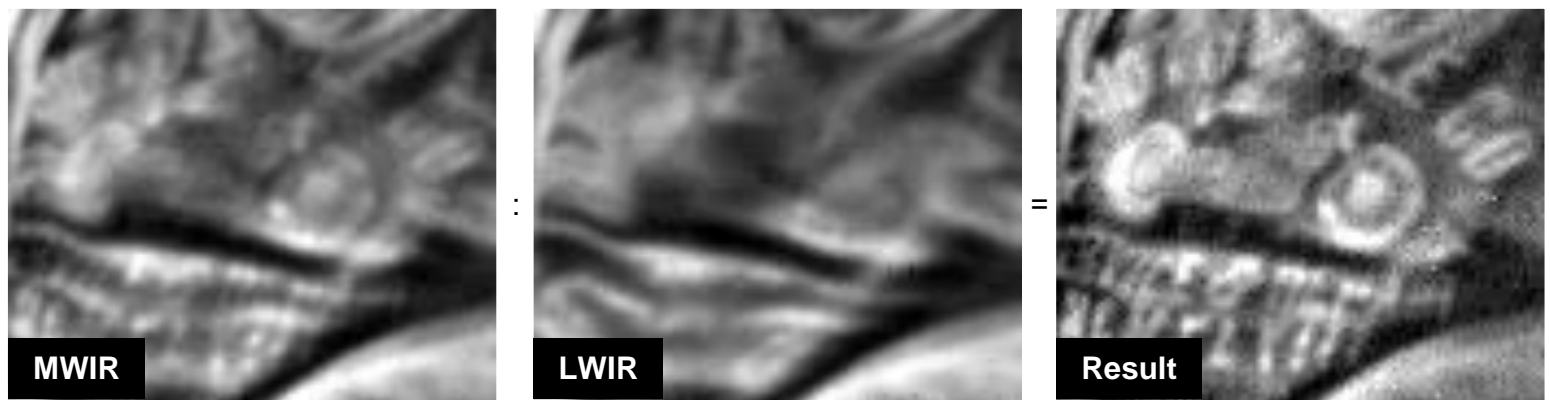

Fig. 7. A T-shirt print taken by passive thermography. Contrast could be enhanced and details revealed by combining the two input images.

\section{Reflectance spectroscopy}

To compare reflection characteristics of CFRP and its components, reflectance was measured with $\mathrm{A}_{2}$ (today Agilent) Technologies Exoscan instrument, a portable FTIR spectrometer, and $45^{\circ}$ external reflection probe attached. Background correction with a reference sample is required by the measurement and was done with a diffuse gold standard disk provided with the instrument. The CFRP sample used for induction thermography was measured at painted and non-painted area. Cured epoxy was measured at the edges of CFRP samples, where no fibres exist. The available area is twice as large as sensor diameter. So the epoxy is the same in thickness and composition as intended during manufacturing for the whole CFRP but side effects and reflections from the edge are possible. Measured areas were flat and smooth. The non-impregnated preform of woven carbon fibres rather turned out to be flexible onto the probe tip. In fact, the high standard deviation could be caused by different air gaps or angles between fibres and sensor. Results are expressed in figure 8 as mean value and standard deviation for measurements at five different positions of the same sample (CFRP painted, non-painted and cured epoxy) or five measurements of different samples (woven carbon fibres). As expected, the black painted CFRP has the least reflection. It is noticeable that the non-painted combination of epoxy resin and carbon fibres reflects more IR radiation than its single components. Other heterogeneous epoxy-based materials show comparable behaviour [8]. Additional interfaces in the three component system of carbon, epoxy and air (e.g. gaps) and a change of scattering because of refractive index ratio are a possible explanation.

Own measurements of reflectance as well as measurements of directional spectral emissivity in literature [3] proved that infrared properties do not vary much up to an angle of $45^{\circ}$. An unknown quantity is the gold standard the measured reflectance in this work is referred to. Nevertheless, mean values measured and from literature for the detected wavebands are summarized in table 1.

Table 1. Summary of reflectance values

\begin{tabular}{|l|c|c|c|c|}
\hline Component & \multicolumn{2}{|c|}{ Reflectance measured [\%] } & \multicolumn{2}{c|}{ Reflectance from literature [\%] } \\
\hline & MWIR & LWIR & MWIR & LWIR \\
\hline CFRP & 20 & 23 & $16[3]$ & $11[3]$ \\
\hline CFRP painted black & 11 & 16 & Highly depending on coating [9] \\
\hline Carbon fibres & 14 & 19 & \multicolumn{2}{|c|}{ Highly depending on configuration } \\
\hline Cured epoxy & 16 & 20 & $\begin{array}{c}13(7.5-13 \mu \mathrm{m})[8] \\
\text { highly depending on composition }\end{array}$ \\
\hline
\end{tabular}




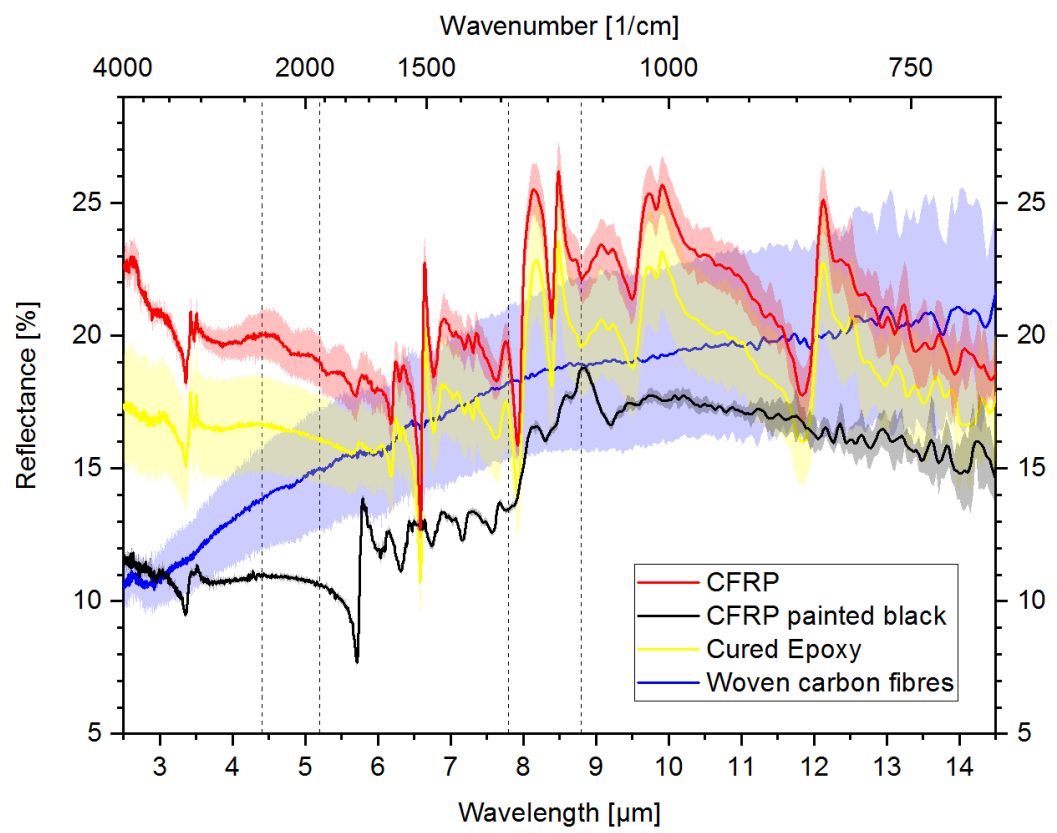

Fig. 8. Reflection of investigated CFRP sample and its components measured by $45^{\circ}$ external reflectance. The standard deviation is shown as shaded area around the curves. Additionally, detection range (full width at half maximum) of MWIR and LWIR infrared detector are marked.

\section{Discussion}

Observations from thermography that the CFRP components can be distinguished better in MWIR than in LWIR detection wave band in areas where reflection is large as well as in areas where reflection is lower than emission (figure 5,6 ) could not be supported by reflectance spectroscopy measurements. The difference in reflectance between carbon fibres and epoxy was small compared with measurement accuracy. It is also possible that absorption and remission of the epoxy layer on the first woven carbon layer highly influence the CFRP spectral properties of infrared emission and reflection. Emissivity $\varepsilon$ is a material and surface property that can be calculated under the assumptions made according to Eq. (2) and is approximated to be constant within detection wave bands. Supposing a surface reflecting less radiation than emitting, the irradiance ratio of two input images taken in different detection wave bands (Eq. (6)) is dominated by an emissivity and a local temperature dependent ratio as follows:

$$
E_{\text {Result }} \approx \frac{\varepsilon_{M W I R}}{\varepsilon_{L W I R}} \cdot \frac{E_{M W I R}(T)}{E_{L W I R}(T)}
$$

High emissivities are essential for the ability to suppress ambient reflections and narcissus effect what could not be observed on surfaces with metallic gloss in additional measurements. Images of textiles and CFRPs could be well improved to reproduce a high dynamic range of luminosity keeping details in highlights and shadows. The method worked like a background correction but without a priori information about structure size as it is needed for common image enhancement like unsharp masking. Because of thermodynamic reason, neighbouring pixels have nearly the same temperature as heat diffuses continuously, whereas materials or surface properties often change abruptly within a two pixel distance in infrared images. Therefore, local contrast of material structure (e.g. emissivity) normally should be emphasized by irradiance ratio. Additionally, the Planck curve plays a significant role as shown in table 2 . Two images with high temperature contrast are processed to an image with high material contrast using Eq. (7). As Watson summarizes, "the spectral ratio method is based on the concept that although the spectral radiances are very sensitive to small changes in temperature the ratios are not." [4]

Table 2. Quantitative change of material and temperature dependant terms in irradiance measurement (neglecting reflection, $E$ for black body) resulting in a contrast between pixels 2 and 1 .

\begin{tabular}{|c|cc|c|c|c|}
\hline Contrast & Term & MWIR & LWIR & MWIR/LWIR \\
\hline Material & $\frac{\varepsilon_{2}-\varepsilon_{1}}{\varepsilon_{1}}$ & $T=$ const. & $12,5 \%$ & $0 \%$ & $12,5 \%$ \\
\hline Temperature & $\frac{E_{2}\left(25^{\circ} \mathrm{C}\right)-E_{1}\left(20^{\circ} \mathrm{C}\right)}{E_{1}\left(20^{\circ} \mathrm{C}\right)}$ & $\varepsilon=$ const. & $18,5 \%$ & $10,5 \%$ & $7,3 \%$ \\
\hline
\end{tabular}




\section{Summary}

Infrared optical properties of carbon-fibre reinforced polymers proved highly wavelength dependant. An irradiance ratio between irradiance of two different wavelengths has been formed to get an emissivity image. The irradiance ratio proved able to suppress reflections and enhance material contrast in thermographic images taken of materials like CFRP or other textiles which is useful in visual and automated inspection. Beside non-destructive testing situations one can think of real-time measurements for machine vision. To get the pixelwise irradiance for every picture, a calibration method has been developed. Based on Planck's law it is flexible to adapt to different wave-bands and only needs two calibration points to get the benefit.

\section{Acknowledgment}

The research leading to these results has received funding from European Regional Development Fund.

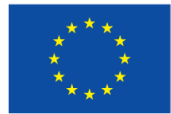

\section{EUROPEAN UNION}

European Regional Development Fund

\section{REFERENCES}

[1] Abuhamad M., "Spektrale Information in der Thermographie" (spectral information in thermography). Doctoral Thesis. Saarland University, Saarbrücken, 2011. DOI: 10.22028/D291-22709.

[2] Abuhamad M, Netzelmann U, "Dual-Band Active Thermography on Infrared Transparent Materials". Quantitative InfraRed Thermography Journal 7 (2), pp. 189-200, 2010. DOI: 10.3166/qirt.7.189-200

[3] Adibekyan A., Kononogova E., Monte C., Gutschwager B., Hollandt J., "Emissivity, reflectivity and transmissivity of semitransparent fibre reinforced plastic composites". In: 13. Thermographie-Kolloquium 2017, Deutsche Gesellschaft für zerstörungsfreie Prüfung (DGZfP) (DGZfP-Berichtsbände, BB 163), Berlin, 2017.

[4] Watson K., "Spectral ratio method for measuring emissivity". Remote Sensing of Environment, Volume 42, Issue 2, pp. 113-116, 1992. DOI: 10.1016/0034-4257(92)90094-Z.

[5] Pron H., Bouache T., "Alternative thermal calibrations of focal plane array infrared cameras." Quantitative InfraRed Thermography Journal 13 (1), pp. 94-108, 2016. DOI: 10.1080/17686733.2015.1131854.

[6] Evangelos T., Juntaro I., Nigel P. F., "A comparison of the performance of a photovoltaic HgCdTe detector with that of large area single pixel QWIPs for infrared radiometric applications". Infrared Physics \& Technology, Volume 46, Issue 4, 2005, pp. 309-322, DOI: 10.1016/j.infrared.2004.05.002.

[7] Berger D., et al., "Effects of defects in series production of hybrid CFRP lightweight components - Detection and evaluation of quality critical characteristics". Measurement, 95, pp. 389-394, 2017.

[8] Houtz D. A., Gu D., "A Measurement Technique for Infrared Emissivity of Epoxy-Based Microwave Absorbing Materials". IEEE Geoscience and Remote Sensing Letters, vol. 15, no. 1, pp. 48-52, Jan. 2018. DOI: 10.1109/LGRS.2017.2772783

[9] Pompea S. M., Breault R. P., "Black surfaces for optical systems". In: Bass M. (Editor in Chief), Handbook of optics, Volume I: Fundamentals, Techniques and Design. 2. ed., chapter 37. Optical Society of America. McGraw-Hill, New York, 1995. 